\title{
A avaliação de programas de saúde: continuidades e mudanças
}

\author{
Health programs assessment: continuities \\ and changes
}

La evaluación de programas de salud: continuidades y cambios

Lígia Maria Vieira-da-Silva 1

Juarez Pereira Furtado 2

doi: 10.1590/0102-311X00237219

\section{Introdução}

A avaliação de programas de saúde tem sido desenvolvida como um conjunto de saberes e práticas com influência de diversas disciplinas científicas e tradições teórico-metodológicas. Tendo em vista o envolvimento de agentes oriundos de diversos campos sociais 1, pode ser considerada um espaço intercampos, no sentido de Bourdieu 2. As trajetórias e posições ocupadas pelos autores têm produzido historicamente diferentes tipos de abordagem e polissemia conceitual 3,4,5. Há ênfases distintas nos aspectos teórico-metodológicos, na prestação de contas e no compromissos com a gestão, dentre outras questões, gerando debates duradouros e mesmo polarizações, sobretudo na produção especializada dos Estados Unidos, do Canadá e da Inglaterra, que concentram certas tradições e significativa produção prática e teórica da área 6 . A delimitação de seu escopo comporta algum grau de arbitrariedade, tendo em vista que políticas, serviços, tecnologias e práticas de saúde 7,8 , sob a denominação de avaliação de programas, têm sido tomados por objeto.

No Brasil, o desenvolvimento da avaliação em saúde ocorreu no interior do campo da Saúde Coletiva 9 e ali se beneficiou de diversas influências ${ }^{1}$. Trabalhos anteriores buscaram identificar convergências e diferenças entre os desenhos da pesquisa avaliativa e da epidemiologia 10, a avaliação de programas e tecnologias 11 , entre as chamadas abordagens estruturadas e não estruturadas 12 , e também entre a avaliação e a análise de políticas ${ }^{13}$. Análises comparativas a esse respeito, entre Brasil e Estados Unidos, mostram o expressivo crescimento das publicações sobre avaliação em saúde a partir dos anos 2000 14, voltadas principalmente para a avaliação de políticas e programas do Sistema Único de Saúde (SUS) 15.

Contudo, um aspecto pouco explorado tem sido a superposição de objetos, conceitos e metodologias que por vezes não estabelecem diálogo entre si. Também a persistência da diversidade de abordagens e modelos tem sido registrada. Em que medida tem havido uma incorporação das proposições teórico-metodológicas dos autores fundadores da disciplina nos trabalhos subsequentes? Em que medida esses modelos têm sido contestados e substituídos ou aperfeiçoados? O presente ensaio pretende contribuir com essas questões e oferecer uma melhor compreensão acerca do desenvolvimento do espaço da avaliação de programas de saúde, buscando atualizar o debate na área, por meio da análise e da discussão de algumas abordagens presentes na produção científica relacionada a essa temática.
1 Instituto de Saúde Coletiva, Universidade Federal da Bahia, Salvador Brasil.

2 Departamento de Politicas Públicas e Saúde Coletiva, Universidade Federal de São Paulo, São Paulo, Brasil.

\section{Correspondência}

L. M. Vieira-da-Silva Instituto de Saúde Coletiva Universidade Federal da Bahia.

Rua Basílio da Gama s/n, Salvador, BA 40110-040, Brasil.

ligiamvs@ufba.br 


\section{Origens da avaliação em saúde como espaço de saberes e práticas (1960-1980)}

Tendo em vista que a avaliação de programas de saúde tem um desenvolvimento muito ligado àquele da avaliação como disciplina acadêmica, uma análise acerca dessa problemática requer algumas considerações preliminares sobre a evolução das relações entre a disciplina da avaliação e os programas de saúde como um dos objeto possíveis.

Nessa perspectiva, pode-se dizer que, na área da saúde, a avaliação passou a ser considerada objeto de estudo e pesquisa a partir de três vertentes que emergiram nos anos 1960. A primeira, oriunda da clínica, estava representada pelas contribuições de Avedis Donabedian 16,17, que sistematizou as diversas abordagens propostas para avaliação da qualidade do cuidado médico. A segunda surgiu no interior da epidemiologia e passou a incorporar como objeto de análise a investigação em serviços de saúde, da qual a avaliação fazia parte 18,19. Já a terceira vertente sofreu a influência das pesquisas avaliativas em educação e em psicologia, iniciadas a partir dos estudos de Campbell \& Stanley 20 e desenvolvidas posteriormente por grupos multidisciplinares reunidos em torno de associações, a exemplo da Fundação Russell Sage (Russell Sage Foundation), em 1967, e da Sociedade de Pesquisa em Avaliação (Evaluation Research Society), em 1978. Entre esses primeiros autores estavam Peter Rossi (Rossi \& Freeman 21), Thomas Cook (Cook \& Campbell 22), Michael Scriven 5 e Carol Weiss 4. Havia também pessoas vinculadas à burocracia governamental, além dos pesquisadores. Inicialmente voltado para o desenvolvimento de uma teoria da avaliação, logo o grupo dividiu-se entre os que compreendiam a avaliação como um guia para a gestão do setor público e aqueles que a concebiam como um recurso para a prestação de contas (accountability). Também se verificou uma especialização com a divisão do espaço dos estudos avaliativos entre disciplinas: educação, políticas públicas e gestão, economia, medicina e saúde pública 23. Esta última vertente foi aquela em que a avaliação foi teorizada de forma mais sistemática e se desenvolveu como disciplina relativamente autônoma. Em coletânea denominada de raízes da avaliação, além dos autores acima mencionados como pioneiros ou fundadores, Christie e Alkin acrescentam Daniel Stufflebeam e mais duas dezenas de autores que, segundo eles, teriam contribuído com teorias ou modelos originais para a disciplina da avaliação 24 .

\section{Algumas tendências na evolução das abordagens para avaliação de programas de saúde (1997-2019)}

Entre 1997 e 2000, surgiram internacionalmente diversas propostas de esquemas ou quadros de referência (frameworks) voltadas para padronizar ou orientar a realização de avaliações de programas de saúde. Algumas foram desenvolvidas como proposições genéricas para a disciplina de avaliação e posteriormente incorporadas às avaliações de programas de saúde, e outras foram especificamente desenvolvidas para essas últimas. Essas propostas fundamentaram diversas avaliações realizadas nas primeiras décadas do século XXI 25,26,27,28,29.

A avaliação de programas denominada realista data de 1997 30, tendo posteriormente evoluído para se apresentar como ciência da avaliação 25. Seu principal formulador, Ray Pawson 25 , situa essa abordagem como parte das ciências sociais, embora defenda a especificidade e autonomia da disciplina da avaliação que permitiriam considerá-la como ciência. Justifica sua formulação, em 1997, em parceria com Tilley, a partir de uma crítica às limitações dos desenhos então dominantes na avaliação que, na sua opinião, ou eram quase experimentais importados dos ensaios clínicos ou descrições formativas de programas locais. A avaliação realista então foi proposta como uma alternativa que buscava explicar a teoria dos programas. A esse respeito, explicita sua filiação à escola denominada de avaliação centrada na teoria (theory-driven evaluation), remetendo sua inspiração à ideia da teoria de médio alcance de Merton e aos trabalhos de Campbell \& Popper 25.

Embora considere que o realismo seja mais uma estratégia de pesquisa geral do que um procedimento técnico, Pawson explicita seus procedimentos metodológicos. Tem por ambição ampliar o objeto da avaliação para além do programa, buscando incorporar o contexto, a história e desvendar os mecanismos das intervenções. O objetivo desse tipo de avaliação seria identificar em um programa "o que funciona, para quem, em quais circunstâncias, a respeito do que, em qual duração (...) e por que" 25 (p. 14). Para isso, propõe analisar o que ele denomina de configuração, contexto, mecanismo e resulta- 
do $(\mathrm{CMOc}) 25$. Contexto seria o conjunto das características dos participantes individuais, suas interrelações, a localização institucional e a infraestrutura ao redor. Mecanismos seriam os processsos, e os resultados seriam os produtos esperados 25. Propugna o uso de múltiplos métodos: evidências qualitativas para analisar os mecanismos, técnicas quantitativas para os resultados e análise histórica para os contextos 25. Embora as semelhanças com a tríade donabediana de estrutura-processo-resultados sejam grandes, o autor não a inclui entre suas referências.

Uma segunda perspectiva corresponde ao esquema analítico proposto por Glasgow et al. 31, em 1999, denominado RE-AIM para avaliação do impacto de intervenções e programas de promoção à saúde. O modelo corresponde à ideia de que o impacto de um programa de Saúde Pública seria uma função de cinco fatores: cobertura, eficácia, adoção, implementação e manutenção (reach, efficacy, adoption, implementation and maintenance). Tendo como objetivo superar as limitações dos ensaios clínicos para avaliação de eficácia e visando a subsidiar as avaliações de efetividade e a pesquisa de disseminação, o RE-AIM reproduz a tradição de alguns estudos precursores relacionada à identificação das principais dimensões para avaliação de um programa de saúde 21. O pressuposto da avaliação dessas dimensões, de forma articulada, é que todas as cinco concorreriam para o impacto de uma política e seriam frequentemente ignoradas em avaliações de eficácia tradicional. No componente eficácia, o modelo inclui tanto efeitos positivos como negativos. Inclui também outros tipos de efeitos, além dos fatores de risco, tanto para os pacientes, a exemplo de comportamentos, como para a equipe de profissionais e sua organização do processo de trabalho. O conceito de adoção está ligado ao número de unidades de saúde que aderem ao programa. Já a efetividade seria um produto da eficácia e da implementação que também é aferida 31. O balanço feito, segundo seus principais idealizadores vinte anos depois, concluiu que houve uma consolidação desse modelo, apoiado nas citações do artigo seminal e em revisão de trabalhos que utilizaram essa metodologia 26.

Há uma terceira abordagem representada por pesquisadores vinculados aos MCR (Conselhos de Pesquisa Médica) da Inglaterra e do Canadá e a diversas universidades inglesas, a exemplo de Oxford e Cambridge. Os membros do conselho de pesquisa em serviços de saúde e saúde pública elaboraram um documento inicial, em 2000, que foi sumarizado em artigo de Campbell et al. ${ }^{32}$. Os principais aspectos do documento relacionam-se ao propósito de avaliação de intervenções complexas por meio de desenhos observacionais. As intervenções complexas, entre outras definições, dizem respeito a ações populacionais. O documento propõe cinco fases para o desenvolvimento de uma avaliação, inspiradas no modelo de estudos sobre medicamentos. Na atualização feita em 2006 a linearidade entre as fases foi criticada, e foi ressaltado pelo comitê que os desenhos experimentais, embora importantes, nem sempre são possíveis. Foi ainda conferida importância ao ajuste das intervenções complexas aos contextos locais 27 .

Numa quarta proposição originada na epidemiologia, o Centro de Controle e Prevenção de Doenças (CDC), vinculado ao Departamento de Saúde e Serviços Humanos do governo americano, publicou um modelo para avaliação por meio do seu programa de epidemiologia 33. A justificativa para a elaboração dessa proposição estava na constatação da ausência de um framework para avaliação em saúde pública, a despeito de toda evolução da avaliação como disciplina. O framework foi elaborado a partir de uma oficina de trabalho envolvendo especialistas em avaliação, gestores em diversos níveis e pesquisadores. Voltado para a incorporação da avaliação na rotina dos serviços de saúde, o modelo do CDC incorpora as etapas de envolvimento dos profissionais responsáveis pelo programa, definição do foco da avaliação e do desenho, elaboração de modelo lógico, entre outras 33 .

Naquele mesmo ano, os epidemiologistas Habicht et al. 34 publicaram um artigo em que analisavam as possíveis contribuições de desenhos alternativos ao experimental para a avaliação de intervenções populacionais. Os autores propuseram um esquema analítico para a escolha da estratégia de investigação sintonizado com a pergunta da avaliação que pode ser considerada como quinta proposição. A abordagem metodológica é composta de dois eixos: o primeiro diz respeito aos indicadores de interesse, a exemplo da utilização de serviços, cobertura ou medidas de impacto. O segundo eixo refere-se ao tipo de inferência a ser feita. A esse respeito, os autores introduzem dois conceitos além da probabilidade para a inferência: adequação e plausibilidade.

Alguns autores que se propõem a realizar avaliações de programas de saúde, a exemplo de Browson et al. 35, situam-se nos desdobramentos da medicina e da saúde pública baseada em evidências que resultaram na denominada pesquisa de implementação e disseminação, da qual a avaliação é um 
capítulo 36. Essa vertente, que partiu da constatação, nos anos 1990, da pequena incorporação da pesquisa nas práticas, tem o propósito de desenvolver-se como uma ciência, da qual a avaliação faria parte. Nessa última perspectiva, há uma tentativa, ainda que retórica, de considerar pertinentes diversas outras abordagens como possibilidades metodológicas para a avaliação voltada para a disseminação e implementação. Com esse objetivo, Gaglio \& Glasgow 37 sintetizaram 11 diferentes modelos para avaliação, entre os quais está a avaliação realista, buscando identificar convergências e especificidades. Ressaltam, contudo, a necessidade de criar pontes entre as abordagens formativas ou de processos e as somativas ou de resultados 37 .

\section{Continuidades e mudanças nas abordagens para avaliação de programas}

Como aspecto em comum entre essas diversas proposições, tem-se a preocupação com a avaliação das intervenções complexas ou da complexidade social como um dos problemas a serem equacionados. Os ensaios randomizados persistem como o contraponto para as abordagens que se propõem a superá-los, a exemplo da avaliação realista e também dos quadros de referência (frameworks) elaborados por grupos de pesquisadores vinculados a instituições de pesquisa, como é o caso do MRC do Reino Unido 38. Outro ponto em comum é o recurso às teorias do comportamento como quadro explicativo para as intervenções a serem avaliadas, bem como a incorporação da noção de contexto. Também é uma constante a articulação entre a avaliação e o momento da intervenção, com o planejamento e com a utilização dos resultados. Nesse sentido, pode-se considerar algumas dessas proposições como um desdobramento e atualização dos estudos clássicos, como os de Cook \& Campbell 22 sobre os desenhos quase experimentais e seu potencial analítico. Destaca-se também a importância da teoria na avaliação desenvolvida por Chen ${ }^{39}$, da utilização dos resultados da avaliação proposta por Patton 40 e até mesmo a preocupação na incorporação do ponto de vista dos participantes (stakeholders) na avaliação, como analisaram Guba \& Lincoln 41 e Fetterman \& Wandersman ${ }^{42}$. Além desses aspectos, nos modelos revisados por Gaglio \& Glasgow 37, foram apontados como pontos convergentes, entre outros, que a avaliação precisa considerar fatores individuais, organizacionais, do staff e da situação, além de questões de custo e custo-efetividade. Contudo, um estudo empírico, a exemplo do que foi feito por Christie 43 sobre o papel de referência teórica na prática dos avaliadores, poderia identificar variações no interior das aparentes concordâncias em torno da concepção e uso de frameworks.

A elaboração de um corpo teórico e metodológico com pretensões à universalidade, revelada pelo uso da ciência como adjetivo conforme proposto por Pawson 25, guardadas as devidas dimensões, pode ser considerada como um movimento na mesma direção daquele, iniciado nos anos 1970, de desenvolvimento de uma teoria da avaliação e de sua autonomização como disciplina específica. Esse esforço tem sido renovado por alguns dos fundadores ainda atuantes e/ou pelos seus seguidores 3 . Por outro lado, a progressiva incorporação da avaliação como componente do processo de implementação das intervenções no desenvolvimento da denominada "ciência" da implementação 44, relacionada às outras áreas com grande intersecção com a pesquisa avaliativa, a exemplo da também rotulada "ciência" dos sistemas de saúde 45 , pode ser considerada como um movimento na direção contrária à autonomização desse espaço social.

As abordagens descritas oscilam entre a pesquisa avaliativa, em que os ensaios randomizados são considerados ainda como desenhos capazes de assegurar maior validade interna e externa, e proposições centradas na utilização e na avaliação formativa, com uso de desenhos pouco estruturados. Entre esses dois polos, haveria um gradiente de proposições que buscariam articular ambas as abordagens, usar métodos mistos, desenvolver desenhos quase experimentais e realizar avaliações centradas na teoria.

Cabe uma breve menção à relação entre alguns estudos brasileiros na área da avaliação e as tendências anteriormente mencionadas. À semelhança do ocorrido nos Estados Unidos e Reino Unido, durante os anos 1990, a avaliação no Brasil desenvolveu-se apoiada nos mesmos referenciais teóricos dos autores das três vertentes referidas na introdução, com uma influência adicional do grupo canadense da Universidade de Montreal 46,47. Por seu turno, o framework desenvolvido pelo grupo canadense incorporava influências de Rossi \& Freeman 21, Chen 39, Patton 40 e Guba \& Lincoln 41 entre outros. Os quadros avaliativos desenvolvidos por autores brasileiros 48,49,50 incorporam alguns 
elementos do modelo canadense, em particular a ênfase nas análises de implantação, mas também desenvolvem esquemas avaliativos semelhantes ao RE-AIM de Glasgow, concebidos praticamente ao mesmo tempo 10,49,51. Essa concomitância também ocorreu no que diz respeito à incorporação das abordagens qualitativas e aos métodos mistos no Brasil 52 e abordagens construtivistas 53 .

\section{Comentários finais}

A análise da evolução dessas abordagens selecionadas sobre a avaliação de programas de saúde revela uma tendência à proposição de novos quadros de referência (frameworks) para avaliação que, por vezes, não dialogam entre si e frequentemente recuperam elementos dos antecessores, sem estabelecer claramente a originalidade das proposições em relação às anteriores.

Dois movimentos aparentemente opostos foram identificados: por um lado, a busca de autonomização da avaliação como ciência; por outro, a sua incorporação em outras disciplinas. A avaliação de programas tanto aparece como uma area temática específica, com problemática e metodologia próprias, quanto como um dos objetos possíveis da temática da avaliação em saúde ao lado da avaliação de políticas, tecnologias e serviços de saúde. Todos esses objetos podem ser avaliados a partir da deliminação do objetivo da avaliação, que pode ser de eficácia, efetividade, qualidade, implantação, acesso entre outras 3,7 .

A diversidade verificada pode ser produto da constituição de um espaço social com pequena autonomia relativa, em que o que está em jogo é a autoridade sobre as questões teórico-metodológicas da avaliação de programas de saúde, não apenas do ponto de vista do conhecimento mas também como mercado de consultorias. É um espaço social que, embora tenha surgido no interior da Saúde Pública, no âmbito internacional, e no campo da Saúde Coletiva no Brasil, é, ao mesmo tempo, dominado pelo campo médico e pelo espaço mais geral da avaliação de programas.

Embora a referência direta aos clássicos da avaliação esteja bastante reduzida em artigos dos últimos cinco anos, ela é mediada por esse conjunto de estudos do final dos anos 1990 e início do século XXI, acima sumarizados, cujos modelos teórico-metodológicos (frameworks) para avaliação de programas de saúde se apoiaram nos autores fundadores da avaliação como disciplina. Pode-se dizer que tanto a avaliação focada na utilização como as abordagens construtivistas foram englobadas nas duas vertentes que buscam a unificação da disciplina acadêmica. Assim, a implementation science incorpora o discurso da avaliação para a utilização e a proposta de uma ciência realista da avaliação, incorporando a teoria e as abordagens qualitativas.

Ainda que a relação da pesquisa avaliativa com as pesquisas em outras disciplinas, particularmente a pesquisa social, seja reconhecida pela maioria dos autores, há uma tendência ambivalente e recorrente de defini-la como uma disciplina autônoma e com especificidades. A proposição de Pawson de uma ciência da avaliação é relativamente recente e passível de críticas que fogem ao escopo desse artigo, porém podem ser indicadas. A principal crítica é a subordinação da avaliação às disciplinas das ciências humanas e sociais que, até o momento, não dispõem de uma teoria unificada do social, e sim, de tradições teóricas 54 . Outro aspecto não menos relevante é a ausência de diálogo com as teorias sociológicas das práticas sociais clássicas e contemporâneas para explicar os programas de saúde e sua implantação.

Dessa forma, a busca da consolidação da avaliação como campo de saberes e práticas relativamente autônomo, no sentido usado por Bourdieu 55 , tem ocorrido concomitantemente com a persistência da tendência histórica à fragmentação e pulverização das abordagens e frameworks, bem como de sua incorporação a outras disciplinas. A dinâmica entre esses dois movimentos pode ser produto não só da composição desse espaço social situado entre os campos científico e burocrático, entre a academia e os serviços de saúde, mas também da natureza do objeto da avaliação, que implica julgamento sobre políticas, governos e governantes 56 . A avaliação de programas de saúde como componente desse espaço social mais amplo sofre influência dessas mesmas tendências. A realização de revisões sistemáticas sobre a temática poderá dimensionar a magnitude dessas diferentes propostas teórico-metodológicas, bem como identificar outras. Também uma análise das trajetórias dos fundadores e autores mais influentes, das redes de relações e das condições históricas de possibilidade poderá contribuir para uma melhor compreensão desses processos. 


\section{Colaboradores}

L. M. Vieira-da-Silva concebeu a problemática do artigo, contribuiu para a revisão bibliográfica, analisou dados e redigiu o manuscrito. J. P. Furtado contribuiu com a construção da problemática, realizou a revisão bibliográfica, analisou dados e participou da redação.

\section{Informações adicionais}

ORCID: Lígia Maria Vieira-da-Silva (0000-00032518-411X); Juarez Pereira Furtado (0000-00016605-1925).

\section{Referências}

1. Furtado JP, Vieira-da-Silva LM. A avaliação de programas e serviços de saúde no Brasil enquanto espaço de saberes e práticas. Cad Saúde Pública 2014; 30:2643-55.

2. Bourdieu P. Sociologie generale. Paris: Seuil; 2016.

3. Alkin MC. Evaluation roots: a wider perspective of theorists' views and influences. 2nd Ed. Los Angeles: Sage; 2013.

4. Weiss CH. Evaluation research methods for assessing program effectiveness. New York: Prentice Hall; 1972.

5. Scriven M. Evaluation thesaurus. 4th Ed. Thousand Oaks: Sage; 1991.

6. Shadish Jr. WR, Cook TD, Leviton LC. Foundations of program evaluation: theories of practice. Newbury Park: Sage; 1999.

7. Rossi PH. My views of evaluation and their origins. In: Alkin MC, editor. Evaluation roots: a wider perspective of theorists' views and influences. 2nd Ed. Los Angeles: Sage; 2013. p. 10612.

8. Mertens DM, Wilson AT. Program evaluation: theory and practice. 2nd Ed. New York: The Guilford Press; 2019.

9. Vieira-da-Silva LM. O campo da Saúde Coletiva: gênese, transformações e articulações com a reforma sanitária brasileira. Rio de Janeiro: Editora Fiocruz/Salvador: EdUFBA; 2018.

10. Vieira-da-Silva LM, Formigli VLA. Avaliação em saúde: limites e perspectivas. Cad Saúde Pública 1994; 10:80-91.

11. Novaes HMD. Avaliação de programas, serviços e tecnologias em saúde. Rev Saúde Pública 2000; 34:547-9.

\section{Agradecimentos}

Os autores agradecem a Wagner Y. Oda, que contribuiu com a revisão preliminar e elaboração do banco de dados que subsidiou a análise.
12. Hartz ZMA. Institutionalizing the evaluation of health programs and policies in France: cuisine internationale over fast food and sur mesure over ready-made. Cad Saúde Pública 1999; 15:229-60.

13. Furtado JP, Gasparini MFV. Há diferenças entre avaliar e analisar? Ciênc Saúde Colet 2019; 24:2933-8.

14. Crumpton CD, Medeiros JJ, Ferreira VRS, Sousa MM, Najberg E. Avaliação de políticas públicas no Brasil e nos Estados Unidos: análise da pesquisa nos últimos 10 anos. Rev Adm Pública 2016; 50:981-1001.

15. Furtado JP, Campos GWS, Oda WY, Campos RO. Planejamento e avaliação em saúde: entre antagonismo e colaboração. Cad Saúde Pública 2018; 34:e00087917.

16. Donabedian A. Evaluating the quality of medical care. Milbank Mem Fund Q 1966; 44:166206.

17. Donabedian A. Explorations in quality assessment and monitoring volume 1: the definition of quality and approaches to its assessment. Ann Arbor: Health Administration Press; 1980.

18. Goldbaum M. Epidemiologia e serviços de saúde. Cad Saúde Pública 1996; 12 Suppl 2:S95-8.

19. Novaes HMD. Pesquisa em, sobre e para os serviços de saúde: panorama internacional e questões para a pesquisa em saúde no Brasil. Cad Saúde Pública 2004; 20 Suppl 2:S147-57.

20. Campbell DT, Stanlley JC. Experimental and quasi experimental designs for research. Boston: Houghton Mifflin Company; 1963. 
21. Rossi PH, Freeman HE. Evaluation: a systematic approach. 3rd Ed. Beverly Hills: Sage; 1985.

22. Cook TD, Campbell DT. Quasi-experimentation: design and analysis issues for field settings. Boston: Houghton Mifflin; 1979.

23. Mark MM, Caracelli V, McNall MA, Miller RL. The oral history of evaluation. Am J Eval 2018; 39:290-304.

24. Christie CA, Alkin MC. An evaluating theory tree. In: Alkin MC, editor. Evaluation roots: a wider perspective of theorists' views and influences. 2nd Ed. Los Angeles: Sage; 2013. p. 1157.

25. Pawson R. The science of evaluation: a realist manifesto. London: Sage; 2013.

26. Glasgow RE, Harden SM, Gaglio B, Rabin B, Smith ML, Porter GC, et al. RE-AIM planning and evaluation framework: adapting to new science and practice with a 20 -year review. Front Public Health 2019; 7:64.

27. Craig P, Dieppe P, Macintyre S, Michie S, Nazareth I, Petticrew M. Developing and evaluating complex interventions: the new Medical Research Council guidance. BMJ 2008; 337:a1655.

28. Rivera Dommarco JA, González de Cosío T, García-Chávez CG, Colchero MA. The role of public nutrition research organizations in the construction, implementation and evaluation of evidence-based nutrition policy: two national experiences in Mexico. Nutrients 2019; 11:594.

29. Heitzman M, Weitzel J, Kroll S, Zabler B. Client experiences in a prenatal home visiting program: a prenatal care coordination program evaluation. Public Health Nurs 2019; 36:653-9.

30. Pawson R, Tilley N. Realistic evaluation. London: Sage; 1997.

31. Glasgow RE, Vogt TM, Boles SM. Evaluating the public health impact of health promotion interventions: the RE-AIM framework. Am J Public Health 1999; 89:1322-7.

32. Campbell M, Fitzpatrick R, Haines A, Kinmonth AL, Sandercock P, Spiegelhalter D, et al. Framework for design and evaluation of complex interventions to improve health. BMJ 2000; 321:694-6.

33. Centers for Disease Control and Prevention. Framework For Program Evaluation In Public Health. MMWR 1999; 48(RR-11):1-40.

34. Habicht J, Victora CG, Vaughan JP. Evaluation designs for adequacy, plausibility and probability of public health programme performance and impact. Int J Epidemiol 1999; 28:10-8.

35. Brownson RC, Colditz CA, Proctor EK, editors. Dissemination and implementation research in health: translating science to practice. 2 nd $\mathrm{Ed}$. New York: Oxford University Press; 2018.

36. Chambers D. Foreword. In: Brownson RC, Colditz CA, Proctor EK, editors. Dissemination and implementation research in health: translating science to practice. 2nd Ed. New York: Oxford University Press; 2018. p. vii-ix.
37. Gaglio B, Glasgow RE. Evaluation approaches for dissemination and implementation research. In: Brownson RC, Colditz CA, Proctor EK, editors. Dissemination and implementation research in health: translating science to practice. 2nd Ed. New York: Oxford University Press; 2018. p. 327-56.

38. Medical Research Council. A framework for the development and evaluation of RCTs for complex interventions to improve health. London: Medical Research Council; 2000.

39. Chen HT. Theory-driven evaluations. Beverly Hills: Sage; 1990.

40. Patton MQ. Utilization-focused evaluation. 2nd Ed. Beverly Hills: Sage; 1986.

41. Guba EG, Lincoln YS. Fourth generation evaluation. Newbury Park: Sage; 1989

42. Fetterman DM, Wandersman A. Empowerment evaluation: theories, principles, concepts and steps. In: Fetterman DM, Kaftarian S, Wandersman A, editors. Empowerment evaluation: knowledge and tools for self-assessment, evaluation capacity building, and accountability. Thousand Oaks: Sage; 2015. p. 20-42.

43. Christie CA. What guides evaluation? A study of how evaluation practice maps onto evaluation theory. New Dir Eval 2003; 2003:7-36.

44. Madon T, Hofman KJ, Kupfer L, Glass RI. Public health: implementation science. Science 2007; 318:1728-9.

45. Skochelak SE, Hawkins RE, Lawson LE, Starr SR, Borkan JM, Gonzalo JD. Health systems science. St. Louis: Elsevier; 2017.

46. Brousselle A, Champagne F, Contandriopoulos A-P, Hartz ZMA, organizadores. Avaliação: conceitos e métodos. Rio de Janeiro: Editora Fiocruz; 2011.

47. Contandriopoulos A-P, Champagne F, Denis J-L, Avargues M. L'evaluation dans le domaine de la santé: concepts et méthodes. Rev Epidemiol Santé Publique 2000; (48):517-39.

48. Hartz ZMA, Champagne F, Contandriopoulos A-P, Leal MC. Avaliação do Programa Materno-infantil: análise de implantação em sistemas locais de saúde no Nordeste do Brasil. In: Hartz ZMA, organizadora. Avaliação em saúde: dos modelos conceituais à prática na análise da implantação de programas. Rio de Janeiro: Editora Fiocruz; 1997. p. 89-131.

49. Vieira-da-Silva LM, Hartz ZMA, Chave SCL, Silva GAP, Paim JS. Análise da implantação da gestão descentralizada em saúde: estudo comparado de cinco casos na Bahia, Brasil. Cad Saúde Pública 2007; 23:355-70.

50. Hartz ZMA, Vieira-da-Silva LM, organizadoras. Avaliação em saúde: dos modelos teóricos à prática na avaliação de programas e sistemas de saúde. Rio de Janeiro: Editora Fiocruz; 2005.

51. Vieira-da-Silva LM. Avaliação do processo de descentralização das ações de saúde. Ciênc Saúde Colet 1999; 4:331-9. 
52. Minayo MCS, Cruz Neto O. Triangulación de métodos en la evaluación de programas y servicios de salud. In: Bronfman M, Castro R, organizadores. Salud, cambio social y política: perspectivas desde América Latina. Cor Del Valle: Edamex; 1999. p. 65-80.

53. Furtado JP. Um método construtivista para a avaliação em saúde. Ciênc Saúde Colet 2001; 6:165-81.
54. Bourdieu P, Chamboredon J-C, Passeron J-C, Teixeira GJF. A profissão do sociólogo: preliminares epistemológicas. Petrópolis: Editora Vozes; 1999.

55. Bourdieu P. As regras da arte: gênese e estrutura do campo literário. Lisboa: Presença; 1996.

56. Carol HW. Rooting for evaluation: digging into beliefs. In: Alkin MC, editor. Evaluation roots: a wider perspective of theorists' views and influences. 2nd Ed. Los Angeles: Sage; 2013. p. 13043. 